\title{
Medical Image of the Week: Lung Cancer with Vascular Invasion
}

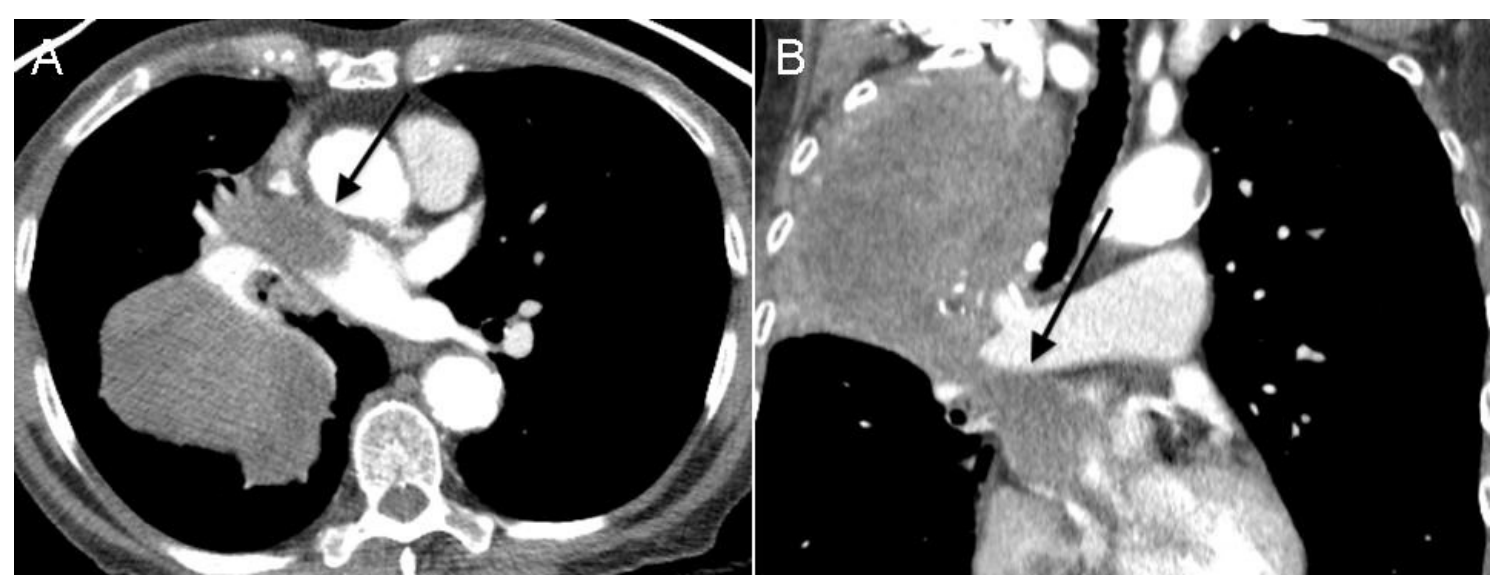

Figure 1. Panel A: Representative axial view of the thoracic CT scan showing tumor invading the left atrium via the right superior pulmonary vein (arrow). Panel B: Coronal view showing tumor invasion (arrow).

A 73-year-old woman presented to the emergency department with seizures and a subacute history of mild dyspnea on exertion. Her admission chest x-ray demonstrated a large right upper lobe lung mass, and MRI of the brain demonstrated multiple bilateral enhancing lesions concerning for a cardiac embolic source. Representative axial (Figure 1A) and coronal (Figure 1B) images from her chest CT scan demonstrate tumor invading the left atrium via the right superior pulmonary vein (arrow). The tumor was confirmed to be small cell carcinoma of the lung.

Joshua Malo, MD and Franz Rischard, DO

Division of Pulmonary, Allergy, Critical Care, and Sleep Medicine University of Arizona, Tucson, AZ 